ISSN 1112-9867

http://www.jfas.info

\title{
SYNTHESIS OF NEW SCHIFF BASES AS MATERIALS FOR THE DESIGN OF PHOTOVOLTAICS CELLS
}

\author{
S. Kasmi-Mir ${ }^{1,2, *}$, M. ElKebich ${ }^{2}$, Z. Dembahri ${ }^{3}$, A. Haouzi ${ }^{2}$ and G. Kirsch ${ }^{4}$ \\ ${ }^{1}$ Laboratoire de Chimie des Substances Naturelles et de Biomolécules, Faculté des Sciences, \\ Université de Blida 1, Algérie \\ ${ }^{2}$ Laboratoire Synthèse et Catalyse, Université Ibn Khaldoun Tiaret, Algérie \\ ${ }^{3}$ Faculté des Sciences, Université de Blida1, Algérie \\ ${ }^{4}$ Laboratoire d'Ingénierie Moléculaire et Biochimie Pharmacologique, EA 3940, Institut Jean \\ Barriol, Université de Lorraine Metz, 1Bld Arago 57070 Metz, France
}

Received: 07 December 2015 / Accepted: 30 April 2016 / Published online: 01 May 2016

\begin{abstract}
We describe the synthesis of new organic Schiff bases chromophores 5 containing a rhodanine-3- acetic as electron accepteur moiety. Imines $\mathbf{3}$ were obtained by a condensation reaction from a lead molecule, the aminothiazolinethione 1 with versatile commercial aldehydes. The structure of all the compounds obtained was determined by the spectroscopic methods (IR, ${ }^{1} \mathrm{H}$ NMR and ${ }^{13} \mathrm{C}$ NMR). The study of UV-Vis imines $\mathbf{3}$ and $\mathbf{5}$ in methanol exhibit the phenomenon of $\pi$ delocalized electrons responsible for the bathochromic effect.
\end{abstract}

Keywords: Aminothiazolinethione; iminomerocyanines; chromophores; rhodanine; bathochrome effect.

Adresse e-mail: kasmi@univ-blida.dz

doi: http://dx.doi.org/10.4314/jfas.v8i2.26

\section{INTRODUCTION}

Face à l'augmentation des besoins énergétiques mondiaux, à l'épuisement des ressources d'énergies fossiles et à la dégradation de l'environnement du au réchauffement climatique, les 
énergies renouvelables font l'objet depuis ces dernières décennies d'une attention grandissante de la part de la communauté scientifique.

L'énergie photovoltaïque qui consiste en la conversion directe de l'énergie solaire en énergie électrique est une des voies les plus prometteuses et s'est considérablement développée aux cours de ces dernières années depuis que le rendement de $24 \%$ a été atteint pour une cellule en silicium cristallin [1]. Afin de diminuer le cout de fabrication des cellules photovoltaïques à base de silicium, la recherche sur les cellules photovoltaïques à partir des matériaux organiques constitue une voie alternative.

La rhodanine, grâce à son caractère fortement attracteur d'électron, a été utilisée dans la synthèse d'une panoplie de colorants organiques pour l'ONL, le solvatochromisme, l'optoélectronique et les cellules solaires [2-6]. Parmi les chromophores à motif rhodanine rapportés dans la littérature, nous citerons les travaux de Morel et ses collaborateurs [7], qui ont montré que les mérocyanines étaient de bonnes candidates pour la préparation des cellules solaires grâce à leur taux de conversion qui était très supérieur à celui des cellules à colorants organiques. Depuis que Grätzel [8] a réalisé un rendement de conversion raisonnable, des efforts considérables ont été entrepris afin d'améliorer le taux de conversion [9]. Plus récemment un colorant nommé D149-indoline (fig 1) comportant la rhodanine acétique en tant qu'attracteur d'électron, a donné une puissance de conversion électrique(PCE) de 9.03\% [10]. L'introduction d'un groupe n-octyle sur le cycle rhodanine du D149-indoline a permis de concevoir un autre D205-indoline [11] qui a amélioré la puissance de conversion électrique d'une valeur de $9.52 \%$.

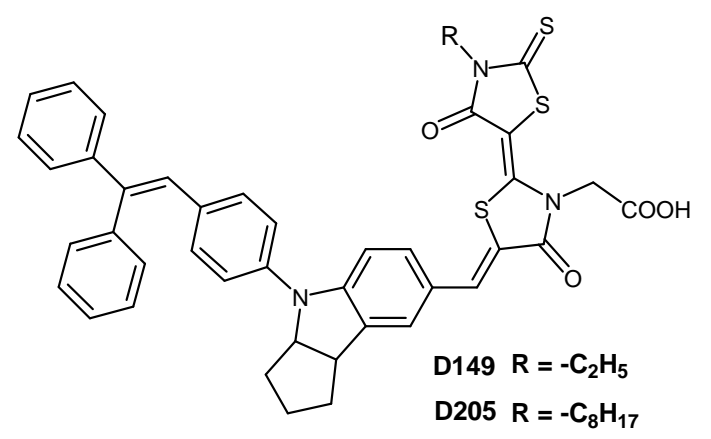

Fig.1. Structures chimiques de D149 et D205

En plus de son implication dans la synthèse des matériaux organiques, la rhodanine et ses dérivés trouvent de nombreuses applications dans le domaine de la biochimie, de la chimie 
médicinale et la chimie des coordinations [12-14].

C'est dans ce contexte de développement des semi-conducteurs organiques pour les applications photovoltaïques que cette étude a été initiée. Elle porte sur la synthèse, la caractérisation structurale de cinq bases de Schiff chromophores, les iminomérocyanines 5 obtenues à partir d'une molécule chef de file, l'aminothiazoline thione 1. Les aldéhydes ont été appliqués sur ce synthon pour aboutir à des imines intermédiaires $\mathbf{2}$ qui renforceront le caractère donneur d'électron de cette dernière. La rhodanine acétique 4 a été introduite comme un attracteur d'électrons

\section{RESULTAS ET DISCUSSIONS}

La réaction de condensation à température ambiante de l'aminothione $\mathbf{1}$ sur différents aldéhydes aromatiques commerciaux dans de l'éthanol et en présence de la pipéridine conduit aisément à la formation d'imine $\mathbf{2}$ (70-92\%). Les sels iminothiazoliums 3 correspondants sont obtenus par une simple alkylation des iminothiazoline thiones $\mathbf{2}$ par un excès d'iodométhane dans de l'acétone (60-81\%) (schéma1).

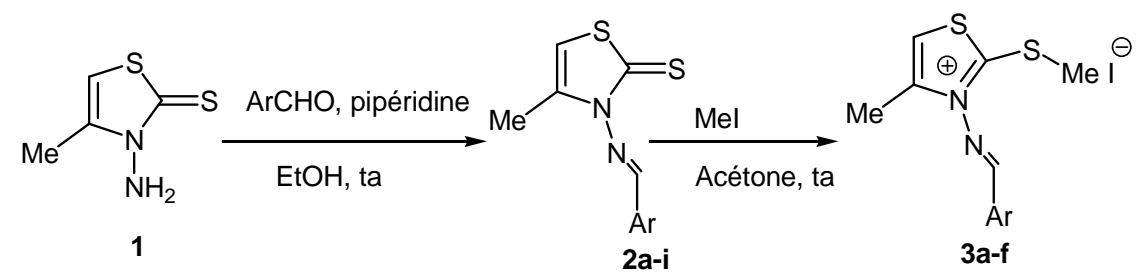

Schéma 1

Tableau 1. Caractéristiques physiques des Iminothiazolinethiones 2a-i

\begin{tabular}{cccc}
\hline Composés & $\mathbf{A r}$ & $\mathbf{R d t \%}$ & Pf $^{\circ} \mathbf{C}$ \\
\hline $\mathbf{2 a}$ & 2-OH- $\mathrm{C}_{6} \mathrm{H}_{4}$ & 90 & 124 \\
$\mathbf{2 b}$ & 4-OMe- $\mathrm{C}_{6} \mathrm{H}_{4}$ & 82 & 130 \\
$\mathbf{2 c}$ & 4-Cl- $\mathrm{C}_{6} \mathrm{H}_{4}$ & 75 & 126 \\
$\mathbf{2 d}$ & Furfuryl & 73 & 78 \\
$\mathbf{2 e}$ & 3-OMe,4-MeO-C $\mathrm{H}_{3}$ & 85 & 150 \\
$\mathbf{2 f}$ & 2-Me- $\mathrm{Indolyl}_{2}$ & 70 & 200 \\
$\mathbf{2 g}$ & $\mathrm{C}_{6} \mathrm{H}_{5}$ & 74 & $>260$ \\
$\mathbf{2 h}$ & 4-N,N-diMe- $\mathrm{C}_{6} \mathrm{H}_{4}$ & 80 & 180 \\
$\mathbf{2 i}$ & 4- $\mathrm{NO}_{2}-\mathrm{C}_{6} \mathrm{H}_{4}$ & 92 & 230 \\
& & & \\
\hline
\end{tabular}


Tableau 2. Caractéristiques physiques des sels iminothiazoliums 3a-f

\begin{tabular}{cccc}
\hline Composés & Ar & Rdt\% & Pf $^{\circ} \mathbf{C}$ \\
\hline 3a & 2-OH- ${ }_{6} \mathrm{H}_{4}$ & 76 & 192 \\
3b & $4-\mathrm{OMe}-\mathrm{C}_{6} \mathrm{H}_{4}$ & 81 & 208 \\
3c & 4-Cl- $\mathrm{C}_{6} \mathrm{H}_{4}$ & 60 & 180 \\
3d & Furfuryl & 75 & 160 \\
3e & 3-OMe,4-MeO- $\mathrm{C}_{6} \mathrm{H}_{3}$ & 72 & 150 \\
3f & 2-Me-Indolyl & 63 & 200 \\
& & & \\
\hline
\end{tabular}

Les caractéristiques physiques des iminothiazolinethiones $\mathbf{2}$ et de leurs sels correspondants $\mathbf{3}$ sont regroupées respectivement dans les tableaux 1 et 2 .

Les bases de Schiff chromophores 5a-e sont obtenues par un couplage entre l'hétérocycle neutre la rhodanine acétique $\mathbf{4}$ et l'hétérocycle cationique $\mathbf{3}$ (le sel d'iodure iminothiazolium) comportant un groupe partant le thiométhyle, porté par un carbone électrophile dans de l'acétone et en présence de la triéthylamine (Schéma 2).
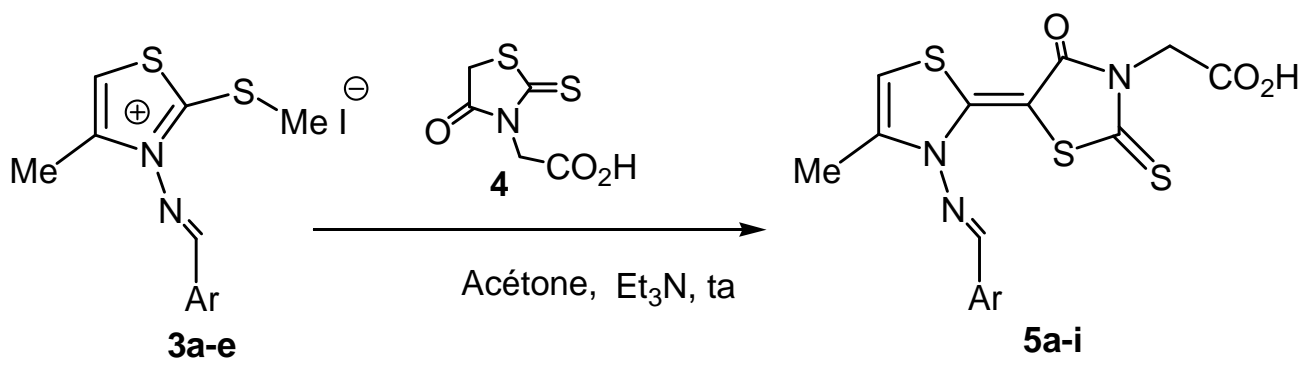

\section{Schéma 2}

Le tableau 3 rassemble les caractéristiques physiques des bases de Schiff chromophores 5.

Tableau 3. Caractéristiques physiques des bases de Schiff chromophores 5a-e

\begin{tabular}{cccc}
\hline Composés & Ar & Rdt\% & Pf $^{\circ} \mathbf{C}$ \\
\hline $5 \mathbf{5 a}$ & $2-\mathrm{OH}-\mathrm{C}_{6} \mathrm{H}_{4}$ & 50 & \\
$\mathbf{5 b}$ & $4-\mathrm{OMe}-\mathrm{C}_{6} \mathrm{H}_{4}$ & 70 & 212 \\
$\mathbf{5 c}$ & $4-\mathrm{Cl}-\mathrm{C}_{6} \mathrm{H}_{4}$ & 45 & 220 \\
$\mathbf{5 d}$ & Furfuryl & 48 & 214 \\
$\mathbf{5 e}$ & 3-OMe,4-MeO-C $6 \mathrm{H}_{3}$ & 76 & 218 \\
& & & 206 \\
\hline
\end{tabular}

L'aminothione $\mathbf{1}$ et la rhodanine acétique $\mathbf{4}$ ont été préparés selon la méthode décrite dans la littérature [15]. 
L'évolution de ces réactions a été suivie par chromatographie sur couche mince.

Les structures des différents composés $\mathbf{1 , 2 , 3 , 4}$ et 5 ont été établies sur la base des données spectrales (IR, $\left.\mathrm{RM}^{1} \mathrm{H},{ }^{13} \mathrm{C}\right)$.

Dans le spectre IR de l'aminothione 1, on observe deux bandes d'absorptions, l'une à 3260 $\mathrm{cm}-1$ et l'autre à $3159 \mathrm{~cm}^{-1}$ caractéristiques de la fonction amine primaire $(\mathrm{N}-\mathrm{H})$. La bande vers $1109 \mathrm{~cm}^{-1}$ est attributable à la fonction thione $\mathrm{C}=\mathrm{S}$. Le spectre de $\mathrm{RMN}^{1} \mathrm{H}$ présente un signal à $2.3 \mathrm{ppm}$ du au groupement méthyle en position 4 couplé avec le proton éthylénique qui apparaît sous forme d'un quadruplet $\left(\mathrm{J}^{4}=1 \mathrm{~Hz}\right)$ vers $6.2 \mathrm{ppm}$; L'apparition d'un signal large vers 4.8ppm correspond aux deux protons de la fonction amine.

Le spectre IR de la rhodanine acétique montre une large bande de vibration d'élongation située vers 3410-2500cm-1, attribuable à la fonction $\mathrm{OH}$ de l'acide et deux bandes de vibration d'élongation située vers $1728 \mathrm{~cm}-1$ et vers $1677 \mathrm{~cm}-1$, correspondant à la fonction $\mathrm{C}=\mathrm{O}$ de la rhodanine et à celle de l'acide carboxylique. En $\mathrm{RMN}$ proton dans $\mathrm{D}_{2} \mathrm{O}$, on observe uniquement deux signaux intégrant deux protons sous forme de singulet, l'un à $4.5 \mathrm{ppm}$ et l'autre à $1.88 \mathrm{ppm}$.

L'identification des imines 2a-i est confirmée en IR par la disparition des deux bandes de vibration $(\mathrm{NH})$ et l'apparition d'une bande vers $1619 \mathrm{~cm}^{-1}$ de la fonction imine $(\mathrm{C}=\mathrm{N})$. La RMN proton indique la présence d'un singulet qui varie entre 9.00 et 9.89ppm attribuable au proton de la fonction imine $(\mathrm{H}-\mathrm{C}=\mathrm{N})$.

Le spectre RMN proton des sels thiazoliums 3a-f montre en plus l'apparition d'un signal sous forme d'un singulet, trés déblindé, vers 2.38-293ppm, caractéristique au thiométhyle $\mathrm{CH} 3-\mathrm{S}$. Ce résultat confirme la méthylation de la fonction thione $\mathrm{C}=\mathrm{S}$.

Le spectre IR des bases de Schiff 5a-e présente une bande large entre 3400 et $2500 \mathrm{~cm}^{-1}$ caractéristique de la bande $\mathrm{OH}$ de l'acide. Deux bandes apparaissent vers $1730-1720$ et 1695-1675 $\mathrm{cm}^{-1}$ sont caractéristiques des carbonyles $(\mathrm{C}=\mathrm{O})$ de la rhodanine. La $\mathrm{RMN}^{1} \mathrm{H}$ montre l'apparition d'un signal vers 4.34-4.48ppm sous forme de singulet affecté aux hydrogènes $\mathrm{CH}_{2}$ de la rhodanine acétique. Le spectre $\mathrm{RMN}^{13} \mathrm{C}$ met en evidence en particulier un signal vers 188.53-189.06ppm attribuable au carbone $\mathrm{C}=\mathrm{S}$ de la rhodanine et deux autres signaux situés vers $167.83-167.85 \mathrm{ppm}$ et $162.99-163.80 \mathrm{ppm}$ correspondants aux carbonyles 
de la rhodanine acétique.

Les analyses d'absorption dans l'UV-Vis ont été effectuées dans le méthanol pour les composés $2 \mathbf{2 a - e}$ et 5a-e. La concentration était de $210^{-5} \mathrm{M}$ pour tous les échantillons.

Une comparaison des $\lambda$ max d'absorption des imines 2a-e avec leurs bases de Schiff correspondantes 5a-e met en évidence le caractère bathochrome (127-152nm).

Tableau 4. Comparaison des absorptions maximums des composés $\mathbf{2}$ et $\mathbf{5}$

\begin{tabular}{ccc}
\hline Composés 2/5 & $\begin{array}{c}\lambda \text { max (2/5) } \\
(\mathbf{n m})\end{array}$ & $\begin{array}{c}\Delta \boldsymbol{\lambda} \max (\mathbf{5 - 3}) \\
(\mathbf{n m})\end{array}$ \\
\hline & & \\
$\mathbf{2 a} / \mathbf{5 a}$ & $277 / 429$ & 152 \\
$\mathbf{2 b} / \mathbf{5 b}$ & $288 / 431$ & 143 \\
$\mathbf{2 c} / \mathbf{5 c}$ & $277 / 422$ & 145 \\
$\mathbf{2 d} / \mathbf{5 d}$ & $293 / 420$ & 127 \\
$\mathbf{2 e} / \mathbf{5 e}$ & $285 / 430$ & 145 \\
\hline
\end{tabular}

\section{PARTIE EXPERIMENTALE}

\subsection{Matériels et méthodes}

Tous les réactifs ont été utilisés sans purification. Les points de fusion ont été déterminés à l'aide d'un banc Koffler et ne sont pas corrigés. Les chromatographies sur couches minces ont été effectuées sur des plaques de silice Merck 60 F254 et révélées par une lampe UV ou par un bain de sable iodé. Les spectres d'absorption infrarouge ont été enregistrés sur un spectromètre Shimadzu 8000 FTIR à l'aide de pastille KBr. Les valeurs des bandes d'absorption sont exprimées en $\mathrm{cm}^{-1}$. Les spectres d'absorptions UV-Vis ont été effectués dans le méthanol $\left(210^{-5} \mathrm{~mol} / \mathrm{l}\right)$ à l'aide d'un appareil Shimadzu 1600. Les spectres RMN ont été enregistrés à l'aide d'un appareil Brücker AC $250(250 \mathrm{MHz}$ pour les protons et 62,9 MHz pour les carbones) au laboratoire LIMBP de Metz de l'Université de la Lorraine.. Les déplacements chimiques $(\delta)$ sont exprimés en parties par million $(\mathrm{ppm})$ par rapport à une réferenceTMS. Les constantes des couplages J sont exprimées en Hertz (Hz). Les abréviations suivantes sont employées: s (singulet); d (doublet); t (triplet); q (quadruplet); Q (quintuplet) m (multiplet). Pour certains composés, malgré des temps d'acquisition et de relaxation importants, les signaux en $\mathrm{RMN}^{13} \mathrm{C}$ ne sont pas visibles. Dans ces derniers cas seuls seront 
indiqués les spectres des protons. L'analyse élémentaire a été effectuée par le laboratoire LIMBP de Metz.

\section{3-2-Synthèse de l'aminothione 1}

Nous avons adopté la sythèse de l'aminothione selon la reference décrite dans la literature [15] avec quelques modifications.

A une suspension d'hydrazine monohydraté $(10 \mathrm{~g}, 0.2 \mathrm{~mol})$ et d'hydroxyde de potassium $(9.35 \mathrm{~g}, 0.16 \mathrm{~mol})$ dans l'éther diéthylique $(100 \mathrm{ml})$, refroidie à $\mathrm{O}^{\circ} \mathrm{C}$, est ajouté goutte à goutte le disulfure de carbone $(10 \mathrm{ml}, 0.16 \mathrm{~mol})$. L'agitation est ensuite maintenue pendant $3 \mathrm{~h}$ à température ambiante. Le monosel de dithiocarbamate de potassium est filtré, puis séché sous vide dans un dessiccateur. Il est utilisé sans purification. Le monosel est ensuite solubilisé dans de l'eau distillé $(150 \mathrm{ml})$ à $\mathrm{O}^{\circ} \mathrm{C}$ sous une vive agitation. La chloroacétone $(15 \mathrm{ml}$, 0.187 moles) est ajoutée en deux fois. L'agitation est maintenue pendant $30 \mathrm{mn}$ à ${ }^{\circ} \mathrm{C}$, puis $3 \mathrm{~h}$ à température ambiante. Le milieu réactionnel est de nouveau refroidi à $\mathrm{O}^{\circ} \mathrm{C}$ avant d'y ajouter quelques gouttes d'acide concentré $\left(\mathrm{HCl}\right.$ ou $\left.\mathrm{H}_{2} \mathrm{SO}_{4}\right)$ pour ramener le $\mathrm{PH}$ à 2 . Il est ensuite porté à reflux pendant $4 \mathrm{~h}$. Après refroidissement, il est hydrolysé par une solution de soude à 10\%. Le précipité obtenu est filtré puis lavé un grand nombre de fois à l'eau glacée. L'aminothione 1 est ensuite recristallisée dans de l'éthanol à $10 \%$.

\section{3-amino-4-methyl-1,3-thiazole-2(3H)-thione 1a}

Cristaux jaunes, Rdt : $35 \%$, Pf : $88^{\circ} \mathrm{C}, \mathrm{Rf}\left(\mathrm{CHCl}_{3}\right): 0.34, \operatorname{IR}(\mathrm{KBr}) \mathrm{cm}^{-1}: 3260(\mathrm{NH}), 3159$ $(\mathrm{NH}), 1109(\mathrm{C}=\mathrm{S}) \cdot \mathbf{R M N}^{1} \mathbf{H}:\left(250 \mathrm{MHz}, \mathrm{CDCl}_{3}\right) \delta \mathrm{ppm}=2.23(\mathrm{~d}, 3 \mathrm{H}, \mathrm{J}=1 \mathrm{~Hz}), 4.8(\mathrm{sl}, 2 \mathrm{H}$, $\left.\mathrm{NH}_{2}\right) ; 6.22(\mathrm{q}, 1 \mathrm{H}, \mathrm{J}=1 \mathrm{~Hz})$. Analyse élémentaire du $\mathrm{C}_{4} \mathrm{H}_{6} \mathrm{~N}_{2} \mathrm{~S}_{2}$ (145.99): \%Calc C32.85; H4.14; N19.16; S43.85;Exp C 32.95; H3.96; N19.57; S44.49.

\subsection{Synthèse des Iminothiazolinethiones $2 \mathrm{a}-\mathrm{f}$}

A 1 éq(10mmoles) d'aminothiazolinethione 1 dissoutes dans un minimum d'éthanol absolu $(10 \mathrm{ml})$, sont ajoutés 1.5 éq(15mmoles) d'aldéhyde et 2 à 3 gouttes de pipéridine. Le mélange réactionnel est mis sous une vive agitation à température ambiante pendant $2 \mathrm{~h}$. Le précipité obtenu est filtré puis séché sous vide.

3-[(2-hydroxybenzylidene) amino]-4-methyl-1,3-thiazole-2(3H)-thione 2a.Cristaux verts. Rdt :90\% ; Pf: $124^{\circ} \mathrm{C} ; \mathrm{Rf}\left(\mathrm{CHCl}_{3}\right)$ 0.52; IR (KBr) cm $\mathrm{cm}^{-1}: 3157$ (O-H) , 3098 ( C=C-H), 1619 
$(\mathrm{C}=\mathrm{N}), 1274(\mathrm{C}=\mathrm{S}) ; \quad \mathrm{UV}-\mathrm{Vis}(\mathrm{MeOH}) \lambda_{\max }: 277 \mathrm{~nm} ; \quad \mathrm{RMN}^{1} \mathrm{H}\left(250 \mathrm{MHz}, \mathrm{CDCl}_{3}\right) \delta \mathrm{ppm}:$ $2.20\left(\mathrm{~d}, 3 \mathrm{H}, \mathrm{J}^{4}=1 \mathrm{~Hz}, \mathrm{CH}_{3}\right), 6.22\left(\mathrm{q}, 1 \mathrm{H}, \mathrm{J}^{4}=1 \mathrm{~Hz}, \mathrm{H}_{5}\right), 6.91-7.44(\mathrm{~m}, 4 \mathrm{Har}),, 9.60(\mathrm{~s}, 1 \mathrm{H}$, $\mathrm{H}-\mathrm{C}=\mathrm{N}), 10.41(\mathrm{sl}, 1 \mathrm{H}, \mathrm{OH}) ; \quad$ Analyse élémentaire du $\mathrm{C}_{11} \mathrm{H}_{10} \mathrm{ON}_{2} \mathrm{~S}_{2}$ (250.02): \%Calc C52.78, H4.03, N11.19, S25.62; Exp C 52.90, H4.50, N11.47, S25.90; SM (Maldi Tof) (m/z): 250.128(M+.), 251.130(M+H), 252.126(M+2H), 253.151(M+3H).

3-[(4-methoxybenzylidene) amino]-4-methyl-1, 3-thiazole-2(3H)-thione 2b. Cristaux verts sous forme d'aiguille. Rdt $82 \%$; Pf: $130^{\circ} \mathrm{C}$; $\mathrm{Rf}\left(\mathrm{CHCl}_{3}\right): 0.53$. IR $(\mathrm{KBr}) \mathrm{cm}^{-1}: 3076(\mathrm{C}=\mathrm{C}-\mathrm{H})$, $1607(\mathrm{C}=\mathrm{N}), 1288(\mathrm{C}=\mathrm{S})$; UV-Vis $(\mathrm{MeOH}) \lambda_{\max }: 288 \mathrm{~nm} \mathrm{RMN}^{1} \mathrm{H}\left(250 \mathrm{MHz}, \mathrm{CDCl}_{3}\right) \delta \mathrm{ppm}$ : $2.30\left(\mathrm{~d}, 3 \mathrm{H}, \mathrm{J}^{4}=1.25 \mathrm{~Hz}, \mathrm{CH}_{3}\right), 3.90(\mathrm{~s}, 3 \mathrm{H}, \mathrm{CH} 3 \mathrm{O}), 6.19\left(\mathrm{q}, 1 \mathrm{H}, \mathrm{J}^{4}=1.25 \mathrm{~Hz}\right), 7.01-7.90(\mathrm{~m}$, 4Har), 9.89(s,1H, H-C=N); $\mathrm{RMN}^{13} \mathrm{C}(62.5 \mathrm{MHz}, \mathrm{DMSO}) \delta \mathrm{ppm}: 14.56\left(\mathrm{CH}_{3}\right), 55.55\left(\mathrm{OCH}_{3}\right), 1$ 04.69(C5), 114.60, 124.05, 130.95 (Car), 139.59(C4), 163.15(Car-OMe), 170.13 (C=N),17 8. $49(\mathrm{C}=\mathrm{S})$.

3-[(4-chlorobenzylidene)amino]-4-methyl-1,3-thiazole-2(3H)-thione 2c Poudre jaune foncé. Rdt: 75\%; Pf: $126^{\circ} \mathrm{C} ; \mathrm{Rf}\left(\mathrm{CHCl}_{3}\right): 0.47$; IR $(\mathrm{KBr}) \mathrm{cm}^{-1}: 3110(\mathrm{C}=\mathrm{C}-\mathrm{H}), 1649(\mathrm{C}=\mathrm{N})$, $1218(\mathrm{C}=\mathrm{S})$; UV-Vis $(\mathrm{MeOH}) \lambda_{\max }: 277 \mathrm{~nm} ; \mathrm{RMN}^{1} \mathrm{H}\left(250 \mathrm{MHz}, \mathrm{DMSO}_{\mathrm{d}-6}\right) \delta \mathrm{ppm}: 2.20(\mathrm{~d}, 3 \mathrm{H}$, $\left.\mathrm{J}^{4}=1.25 \mathrm{~Hz}, \mathrm{CH}_{3}\right), \quad 6.83\left(\mathrm{q}, 1 \mathrm{H}, \mathrm{J}^{4}=1.25 \mathrm{~Hz}\right), 7.66-7.98(\mathrm{~m}, 4 \mathrm{Har}), 9.33(\mathrm{~s}, 1 \mathrm{H}, \mathrm{H}-\mathrm{C}=\mathrm{N})$.

3-[(furan-2-ylmethylidene) amino]-4-methyl-1,3-thiazole-2(3H)-thione 2d Cristaux verts. Rdt: $73 \%$; Pf: $78^{\circ} \mathrm{C} ; \mathrm{Rf}\left(\mathrm{CHCl}_{3}\right): 0.49$; IR $(\mathrm{KBr}) \mathrm{cm}^{-1}: 3100(\mathrm{H}-\mathrm{C}=\mathrm{C}), 1210(\mathrm{C}=\mathrm{S})$; UV-Vis $(\mathrm{MeOH}) \lambda_{\max }: 293 \mathrm{~nm} ; \mathrm{RMN}^{1} \mathrm{H}\left(250 \mathrm{MHz}, \mathrm{CDCl}_{3}\right) \delta \mathrm{ppm}: 2.20\left(\mathrm{~d}, 3 \mathrm{H}, \mathrm{J}^{4}=1.25 \mathrm{~Hz}, \mathrm{CH}_{3}\right)$, $6.20\left(\mathrm{q}, 1 \mathrm{H}, \mathrm{J}^{4}=1.25 \mathrm{~Hz}\right), 6.35(2 \mathrm{H}, \mathrm{d}, \mathrm{J}=3.6 \mathrm{~Hz}, 2 \mathrm{H}$ Fur $), 7.70(2 \mathrm{H}, \mathrm{d}, \mathrm{J}=3.6 \mathrm{~Hz})$, 9.33(s,1H,H-C=N).

\section{3-[(3-hydroxy-4-methoxybenzylidene)amino]-4-methyl-1,3-thiazole-2(3H)-thione2e}

Cristaux jaunes. Rdt: $85 \%$; Pf: $150^{\circ} \mathrm{C} ; \quad \mathrm{Rf}\left(\mathrm{CHCl}_{3}\right): 0.45 ; \quad \mathrm{IR}(\mathrm{KBr}) \mathrm{cm}^{-1}: 3160(\mathrm{OH}), 16$ $20(\mathrm{C}=\mathrm{N}), 1277(\mathrm{C}=\mathrm{S})$; UV-Vis $(\mathrm{MeOH}) \lambda_{\max }: 285 \mathrm{~nm} ; \quad \mathrm{RMN}^{1} \mathrm{H}\left(250 \mathrm{MHz}, \mathrm{CDCl}_{3}\right) \delta \mathrm{ppm}$ : $2.27\left(\mathrm{~d}, 3 \mathrm{H}, \mathrm{J}^{4}=1.25 \mathrm{~Hz}\right) ; 3.97\left(\mathrm{~s}, 3 \mathrm{H}, \mathrm{J}^{4}=1.25 \mathrm{~Hz}\right) ; 6.99-7.56(\mathrm{~m}, 3 \mathrm{Har}) ; 9.28(\mathrm{~s}, 1 \mathrm{H}, \mathrm{OH})$.

(E)-4-methyl-3-((2-methylindolin-3-yl)methyleneamino)thiazole-2(3H)-thione2f. Poudre jaune. Rdt: $70 \%$; Pf: $200^{\circ} \mathrm{C} ; \mathrm{Rf}\left(\mathrm{CHCl}_{3}\right): 0.25 ; \mathrm{RMN}^{1} \mathrm{H}\left(250 \mathrm{MHz}, \mathrm{CDCl}_{3}\right) \delta \mathrm{ppm}: 1.56 \mathrm{~b}(\mathrm{~d}$, $\left.3 \mathrm{H}, \mathrm{J}^{4}=1.25 \mathrm{~Hz}, \mathrm{CH}_{3}\right), 6.22\left(\mathrm{q}, \mathrm{J}^{4}=1.25 \mathrm{~Hz}, \mathrm{H}_{5}\right), 7.22-7.50(\mathrm{~m}, 4 \mathrm{Har}), 9.57(\mathrm{~s}, 1 \mathrm{H}, \mathrm{OH}), 10.24$ (sl,1H, NH); $\left.\mathrm{RMN}^{13} \mathrm{C}\left(62.5 \mathrm{MHz}, \mathrm{DMSO}_{\mathrm{d}-6}\right) \delta \mathrm{ppm}: 11.40\left(\mathrm{CH}_{3}\right) \mathrm{Thiazol}\right)+\mathrm{CH}_{3}$ indol $), 111.32$ 
$\left(\mathrm{C}_{5}\right), 113.58,119.91,121.79,122.55,125.52(\mathrm{Car}), 135.28(\mathrm{C} 4), 159.09(\mathrm{C}=\mathrm{N}), 184.13(\mathrm{C}=\mathrm{S})$.

3-(benzylideneamino)-4-methyl-1,3-thiazole-2(3H)-thione 2g. Cristaux jaunes. Rdt: 74\%; Pf: >260 ${ }^{\circ} \mathrm{C}$; Rf $\left(\mathrm{CHCl}_{3}\right): 0.40$; IR $(\mathrm{KBr}) \mathrm{cm}^{-1}: \quad 1609(\mathrm{C}=\mathrm{N}), 1210(\mathrm{C}=\mathrm{S}) ; \mathrm{RMN}^{1} \mathrm{H}(250 \mathrm{MHz}$, $\left.\mathrm{CDCl}_{3}\right) \delta \mathrm{ppm}: 1.59\left(\mathrm{~d}, 3 \mathrm{H}, \mathrm{J}^{4}=1.00 \mathrm{~Hz}, \mathrm{CH}_{3}\right), 6.18\left(1 \mathrm{H}, \mathrm{q}, \mathrm{J}^{4}=1.00 \mathrm{~Hz}, \mathrm{H}_{5}\right), 7.20-7.86(\mathrm{~m}$, 4Har), 9.00(s, 1H, H-C=N).

\section{3-\{[4-(dimethylamino)benzylidene]amino\}-4-methyl-1,3-thiazole-2(3H)-thione $2 \mathrm{~h}$}

Cristaux jaune dorés. Rdt: $80 \%$. Pf: $180^{\circ} \mathrm{C} . \operatorname{Rf}\left(\mathrm{CHCl}_{3}\right): 0.25$. IR $(\mathrm{KBr}) \mathrm{cm}^{-1}: 3095(\mathrm{H}-\mathrm{C}=\mathrm{C})$; $1584(\mathrm{C}=\mathrm{N}) ; 1180(\mathrm{C}=\mathrm{S}) . \mathrm{RMN}^{1} \mathrm{H}\left(250 \mathrm{MHz}, \mathrm{CDCl}_{3}\right) \delta \mathrm{ppm}=2.25\left(\mathrm{~d}, 3 \mathrm{H}, \mathrm{J}^{4}=1.00 \mathrm{~Hz}, \mathrm{CH}_{3}\right)$, $3.1\left(\mathrm{~s}, 6 \mathrm{H}, \mathrm{N}\left(\mathrm{CH}_{3}\right)_{2}\right) ; \quad 6.22\left(\mathrm{~d}, 1 \mathrm{H}, \mathrm{J}^{4}=1.00 \mathrm{~Hz}, \mathrm{H}_{5}\right), 6.90-7.90(\mathrm{~m}, 4 \mathrm{Har}, \mathrm{J}=1.00 \mathrm{~Hz}), 9.2(\mathrm{~s}$, $1 \mathrm{H}, \mathrm{H}-\mathrm{C}=\mathrm{N})$.

4-methyl-3-[(4-nitrobenzylidene)amino]-1,3-thiazole-2(3H)-thione 2i Cristaux rouges. Rdt: 92\%; Pf: $230^{\circ} \mathrm{C}$; $\mathrm{Rf}\left(\mathrm{CHCl}_{3}\right): 0.50 ; \mathrm{IR}(\mathrm{KBr}) \mathrm{cm}^{-1}: 3106(\mathrm{C}=\mathrm{C}-\mathrm{H}), 1518(\mathrm{C}=\mathrm{N})$, $1178(\mathrm{C}=\mathrm{S}) ; \mathrm{RMN}^{1} \mathrm{H}\left(250 \mathrm{MHz}, \mathrm{CDCl}_{3}\right) \delta \mathrm{ppm}: 2.26\left(\mathrm{~d}, 3 \mathrm{H}, \mathrm{J}^{4}=1.25 \mathrm{~Hz}, \mathrm{CH}_{3}\right), 6.79(\mathrm{q}, 1 \mathrm{H}$, $\left.\mathrm{J}^{4}=1.25 \mathrm{~Hz}\right), 8.18-8.37(\mathrm{~m}, 4 \mathrm{Har}), 9.75(\mathrm{~s}, 1 \mathrm{H}, \mathrm{H}-\mathrm{C}=\mathrm{N})$.

\subsection{Synthèse de sels des iminothiazoliums 3}

Un mélange de 1éq (5mmoles) d'imine 2 avec un excès d'iodométane 3éq (15mmoles) est mis dans l'acétone sous une vive agitation et à température ambiante jusqu'à formation du sel.

Iodure de 3-\{[(Z)-(2-hydroxyphenyl)methylidene]amino\}-4-methyl-2 (methylsulfanyl) -1,3-thiazol 3a. Cristaux blancs. Rdt: 76\%; Pf: $192^{\circ} \mathrm{C}$; IR (KBr) cm ${ }^{-1}: 3162(\mathrm{O}-\mathrm{H}), 1599$ $(\mathrm{C}=\mathrm{N}) ; \mathrm{RMN}^{1} \mathrm{H}\left(250 \mathrm{MHz}, \mathrm{DMSO}_{\mathrm{d}-6}\right) \delta \mathrm{ppm}: 2.35$ (d, 3H, J ${ }^{4}=1.25 \mathrm{~Hz}, \mathrm{CH}_{3}$ thiazol), 2.91(s, $\left.3 \mathrm{H}, \mathrm{CH}_{3}-\mathrm{S}\right), 7.20\left(\mathrm{q}, 1 \mathrm{H}, \mathrm{J}=1.25 \mathrm{~Hz}, \mathrm{H}_{5}\right)$, 7.54-7.97. (m, 4Har), 9.31(s, 1H, H-C=N), 10.94(s, $1 \mathrm{H}, \mathrm{OH})$.

Iodure de 3-\{[(E)-(4-methoxyphenyl)methylidene $]$ amino\}-4-methyl-2-(methylsulfanyl)-1,3

-thiazol-3-ium 3b .Cristaux blancs. Rdt: 81\%; Pf: 208 ${ }^{\circ}$; IR (KBr) $\mathrm{cm}^{-1}$ : 3091 (C=C-H), $1599(\mathrm{C}=\mathrm{N})$; RMN1H (250MHz, DMSOd-6) $\delta \mathrm{ppm}=2.39\left(\mathrm{~d}, 3 \mathrm{H}, \mathrm{J}^{4}=1.00 \mathrm{~Hz}, \mathrm{CH}_{3}\right.$ thiazol $)$, 2.91(s, 3H, $\left.\mathrm{CH}_{3}-\mathrm{S}\right), 3.88\left(\mathrm{~s}, 3 \mathrm{H}, \mathrm{CH}_{3} \mathrm{O}\right), 7.20\left(\mathrm{q}, 1 \mathrm{H}, \mathrm{J}^{4}=1.00 \mathrm{~Hz}\right), 7.17-7.95(\mathrm{~m}, 4 \mathrm{H}), 9.06(\mathrm{~s}$, $1 \mathrm{H})$.

Iodure de 3-\{[(E)-(4-chlorophenyl)methylidene]imino\}-4-methyl-2-(methylsulfanyl)-1,3- 
thiazol-3-ium 3c. Cristaux jaunes. Rdt: 60\%; Pf: $180^{\circ} \mathrm{C}$; IR $(\mathrm{KBr}) \mathrm{cm}^{-1}: 3017(\mathrm{C}=\mathrm{C}-\mathrm{H})$, $1592(\mathrm{C}=\mathrm{N}) ; \mathrm{RMN}^{1} \mathrm{H}\left(250 \mathrm{MHz}, \mathrm{CDCl}_{3}\right) \delta \mathrm{ppm}: 2.41\left(\mathrm{~d}, 3 \mathrm{H}, \mathrm{J}^{4}=1.00 \mathrm{~Hz}, \mathrm{CH}_{3}\right), 2.93(\mathrm{~s}, 3 \mathrm{H}$, $\left.\mathrm{CH}_{3}-\mathrm{S}\right), 7.90\left(\mathrm{q}, 1 \mathrm{H}, \mathrm{J}^{4}=1.00 \mathrm{~Hz}, \mathrm{H}_{5}\right), 7.73-8.05(\mathrm{~m}, 4 \mathrm{Har}), 9.33(\mathrm{~s}, 1 \mathrm{H}, \mathrm{H}-\mathrm{C}=\mathrm{N}) ; \mathrm{RMN}^{1} \mathrm{H}$ $\left(62.5 \mathrm{MHz}, \mathrm{CDCl}_{3}\right) \delta \mathrm{ppm}: 13.32\left(\mathrm{CH}_{3}\right), 17.35\left(\mathrm{CH}_{3}-\mathrm{S}\right), 104.94\left(\mathrm{C}_{5}\right.$ thiazol $), 117.03 ; 129.78$; 130.53 (Car) ; 139.76(C $\mathrm{C}_{4}$ thiazol), 142.73(Car-Cl), 169.12(C=N), 174.74(C-S).

Iodure de 3-\{[(E)-furan-2-ylmethylidene ]amino\}-4-methyl-2-(methylsulfanyl)-1,3hiazol-3-ium 3d Cristaux blancs. Rdt: 75\%. Pf: $160^{\circ} \mathrm{C}$. IR $(\mathrm{KBr}) \mathrm{cm}^{-1}: 3019(\mathrm{C}=\mathrm{C}-\mathrm{H})$, $1597(\mathrm{C}=\mathrm{N}) . \quad \mathrm{RMN}^{1} \mathrm{H}:\left(250 \mathrm{MHz}, \mathrm{CDCl}_{3}\right) \delta \mathrm{ppm}=2.23\left(\mathrm{~d}, 3 \mathrm{H}, \mathrm{J}^{4}=1.00 \mathrm{~Hz}, \mathrm{CH}_{3}\right.$ thiazol $)$, 2.89(s, 3H, $\left.\mathrm{CH}_{3}-\mathrm{S}\right), 7.90\left(\mathrm{q}, 1 \mathrm{H}, \mathrm{J}=1.00 \mathrm{~Hz}, \mathrm{H}_{5}\right), 7.73-8.05(\mathrm{~m}, 4 \mathrm{H}), 9.10(\mathrm{~s}, 1 \mathrm{H}, \mathrm{H}-\mathrm{C}=\mathrm{N})$.

Iodure de 3-\{[(E)-(4-hydroxy-3-methoxyphenyl)methylidene]amino\}-4-methyl-2-(methyl sulfanyl)-1,3-thiazol $3 \boldsymbol{e}$ Cristaux jaunes. Rdt: 72\%; Pf: $150^{\circ} \mathrm{C}$; IR (KBr) $\mathrm{cm}^{-1}: 3506(\mathrm{O}-\mathrm{H})$, 3088( C=C-H), $1590(\mathrm{C}=\mathrm{N}) ; \mathrm{RMN}^{1} \mathrm{H}(250 \mathrm{MHz}, \mathrm{DMSOd}-6) \delta \mathrm{ppm}: 2.37$ (d, 3H, J4 = 1.25Hz, $\mathrm{CH} 3$ ), 2.38(s, 3H, $\left.\mathrm{CH}_{3}-\mathrm{S}\right), 3.32$ (s, 3H, $\left.\mathrm{CH}_{3} \mathrm{O}\right), 7.01\left(\mathrm{q}, 1 \mathrm{H}, \mathrm{J}^{4}=1.25 \mathrm{~Hz}, \mathrm{H}_{5}\right.$ thiazol), 7.91 (s, 1 $\mathrm{H}$ ) ,7.42-7.53(m,3Har), 9.95 (s, 1H, H- C =N);10.59(s, $1 \mathrm{H}, \mathrm{OH}) ; \mathrm{RMN}^{13} \mathrm{C}(62.5 \mathrm{MHz}$, DMSOd-6) Sppm: 13.28( $\left.\mathrm{CH}_{3}\right), 17.04\left(\mathrm{CH}_{3}-\mathrm{S}\right), 55.66\left(\mathrm{CH}_{3}-\mathrm{O}\right), 110.82\left(\mathrm{C}_{5}\right.$ thiazol $), 114.46,115$ $.87,116.67,121.15,127.08(\mathrm{Car}), 143.04\left(\mathrm{C}_{4}\right.$ thiazol),148.33,153.82(Car),168.19 (C=N),174.67 (C-S).

4-methyl-3-\{[(E)-(2-methyl-2,3-dihydro-1H-indol-3-yl)methylidene ]imino\}-2-(methylsulfan yl)-1,3-thiazol-3-ium 3f. Cristaux oranges. Rdt: 63\%; Pf: $200^{\circ} \mathrm{C}$; $\mathrm{RMN}^{1} \mathrm{H}\left(250 \mathrm{MHz}, \mathrm{CDCl}_{3}\right)$ $\delta \mathrm{ppm}: 2.06\left(\mathrm{~d}, 3 \mathrm{H}, \mathrm{J}^{4}=1.25 \mathrm{~Hz}, \mathrm{CH}_{3}\right), \quad 2.66\left(\mathrm{~s}, 3 \mathrm{H}, \mathrm{CH}_{3}-\mathrm{S}\right), 3.32\left(\mathrm{~s}, 3 \mathrm{H}, \mathrm{CH}_{3} \mathrm{indol}\right), 7.12$ (q, $\left.1 \mathrm{H}, \mathrm{J}^{4}=1.25 \mathrm{~Hz}\right), 7.35(\mathrm{~m}, 4 \mathrm{Har}), \quad 10.04(\mathrm{~s}, 1 \mathrm{H}, \mathrm{OH}), 11.98(\mathrm{sl}, 1 \mathrm{H}, \mathrm{NH})$.

\subsection{Synthèse de la rhodanine acétique 4}

Nous avons adopté la méthode décrite dans la littérature [15] pour préparer la rhodanine acétique 4 avec quelques modifications. Le monosel obtenu est solubilisé dans de l'eau distillé $(150 \mathrm{ml})$ à $\mathrm{O}^{\circ} \mathrm{C}$ sous une vive agitation. L'acide chloroacétique $(0.25 \mathrm{moles})$ est ajoutée en deux fois. L'agitation est maintenue pendant $20 \mathrm{mn}$ à ${ }^{\circ} \mathrm{C}$, puis $3 \mathrm{~h}$ à température ambiante, le PH doit être acide. Il est ensuite porté à reflux pendant $3 \mathrm{~h}$. Le volume est évaporé sous pression réduite. Le résidu est recristallisé dans un minimum d'éthanol pour conduire à des cristaux jaunes. 
Acide-(4-oxo-2-thioxothiazolidin-3-yl) (rhodanine acétique)4 Cristaux jaunes. .Rdt: 43\%; Pf: $184^{\circ} \mathrm{C}$; IR $(\mathrm{KBr}) \mathrm{cm}^{-1}:$ 3410-2500(O-H) acide $, 1728,1677(\mathrm{C}=\mathrm{O}), 1285(\mathrm{C}=\mathrm{S})$.; $\mathrm{RMN}^{1} \mathrm{H}$ $\left(250 \mathrm{MHz}, \mathrm{D}_{2} \mathrm{O}\right) \delta \mathrm{ppm}: 1.88\left(\mathrm{~s}, 2 \mathrm{H}, \mathrm{H}_{5}\right), 4.5\left(\mathrm{~s}, 2 \mathrm{H}, \mathrm{CH}_{2} \mathrm{CO}\right)$,

\subsection{Synthèse des iminomérocyanines 5a-e}

Dans un ballon contenant $15 \mathrm{ml}$ d'acétone, on place successivement, le sel imine $\mathbf{3}$ (1 $\mathrm{mmol}$ ), la rhodanine $4(1.2 \mathrm{mmol})$ et 3 gouttes de triéthylamine. Le mélange réactionnel est mis sous agitation à température ambiante pendant30mn au bout duquel un solide se forme. Il est recristallisé dans de l'éthanol, puis filtré sur papier filtre et séché sous vide.

Acide\{(5E)-5-[3-\{[(E)-(2-hydroxyphényl)méthylidene]amino\}-4-méthyl-1,3-thiazol-2(3H)-yl idéne]-4-oxo-2-thioxo-1,3-thiazolidin-3-yl\} 5a.Poudre rouge brique. Rdt: 50\%, Pf: $212^{\circ} \mathrm{C}$, $\mathrm{R}_{\mathrm{f}(\mathrm{CHCl} 3 / \mathrm{MeOH}, 9 / 1)}: 0.8$; (MeOH) $\lambda \max : 429 \mathrm{~nm}$; IR (KBr) $\mathrm{cm}^{-1}: 3400-2500($ OHacide) , 1728(C=O), 1677(C=O), 1588(C=N),1191(C=S); $\mathrm{RMN}^{1} \mathrm{H}\left(250 \mathrm{MHz}, \mathrm{DMSO}_{\mathrm{d}-6}\right) \delta \mathrm{ppm}$ : $2.35\left(\mathrm{~d}, 3 . \mathrm{H}, \mathrm{J}^{4}=1.00 \mathrm{H}, \mathrm{CH}_{3}\right), 4.40\left(\mathrm{~s}, 2 \mathrm{H}, \mathrm{CH}_{2} \mathrm{CO}\right), 6.80\left(\mathrm{q}, 1 \mathrm{H}, \mathrm{J}^{4}=1.00 \mathrm{~Hz}, \mathrm{H}_{5}\right.$ thiazol), 7.08-7.69 (m, 4Har), 8.54(s, 1H, OH), 9.30(s, 1H, H-C=N); $\mathrm{RMN}^{13} \mathrm{C}\left(62.5 \mathrm{MHz}, \mathrm{DMSO}_{\mathrm{d}-6}\right) \delta$ ppm: 13.29 $\left(\mathrm{CH}_{3}\right), 49.81\left(\mathrm{CH}_{2}\right), 104.72\left(\mathrm{C}_{5}\right.$ thiazol $), 114.76,122.25,127.45,131.80(\mathrm{Car})$, 136.85(C4thiazol) ,146.72(C-OH) ,160.55(C=N), 162.99(C=O) ,167.83(C=O), 188.60(C=S). Acide\{(5Z)-5-[3-\{[(E)-(4-méhoxyphényl)méthylidéne]amino\}-4-méthyl-1,3-thiazol-2(3H)-yli déne]-4-oxo-2-thioxo-1,3-thiazolidin-3-yl\} 5b. Poudre orange. Rdt: $70 \%$; Pf: $220^{\circ} \mathrm{C} ; \mathrm{R}_{\mathrm{f}}$ (CHCl3/MeOH, 9/1) : 0.6 ; (MeOH) $\lambda \max : 431 \mathrm{~nm}$; IR (KBr) $\mathrm{cm}^{-1}: 3500-2500$ (OHacide), 1728 $(\mathrm{C}=\mathrm{O}),\left(1675(\mathrm{C}=\mathrm{O}), 1582(\mathrm{C}=\mathrm{N}), 1160(\mathrm{C}=\mathrm{S}) ; \mathrm{RMN}^{1} \mathrm{H}\left(250 \mathrm{MHz}, \mathrm{DMSO}_{\mathrm{d}-6}\right) \delta \mathrm{ppm}: 2.23\right.$ $\left(\mathrm{d}, 3 \mathrm{H}, \mathrm{J}^{4}=, 1.00 \mathrm{H}, \mathrm{CH}_{3}\right), 3.07\left(\mathrm{~s}, 3 \mathrm{H}, \mathrm{CH}_{3} \mathrm{O}\right), 4.47\left(\mathrm{~s}, 2 \mathrm{H}, \mathrm{CH}_{2} \mathrm{CO}\right), 6.89\left(\mathrm{q}, 1 \mathrm{H}, \mathrm{J}^{4}=\right.$, $1.00 \mathrm{H}), 7.23-7.99(\mathrm{~m}, 4 \mathrm{Har}), 8.93(\mathrm{~s}, 1 \mathrm{H}, \mathrm{H}-\mathrm{C}=\mathrm{N}) ; \mathrm{RMN}^{13} \mathrm{C}\left(62.5 \mathrm{MHz}, \mathrm{DMSO}_{\mathrm{d}-6}\right) \delta \mathrm{ppm}$ : 13.36 $\left(\mathrm{CH}_{3}\right), 45.63\left(\mathrm{CH}_{3} \mathrm{O}\right), 55.68\left(\mathrm{CH}_{2}\right), 81.02,104.90\left(\mathrm{C}_{5}\right.$ thiazol $), 115.02,123.69,126.28$, $131.71 ; 135.31,150.38,159.13(\mathrm{C}=\mathrm{N}), 163.80(\mathrm{C}=\mathrm{O}), 167.85(\mathrm{C}=\mathrm{O}), 188.53(\mathrm{C}=\mathrm{S})$.

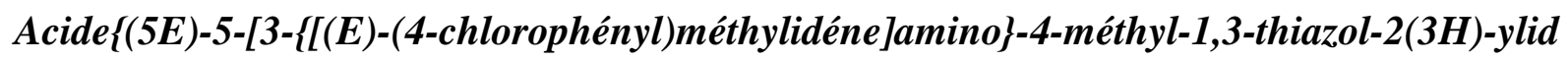
éne]-4-oxo-2-thioxo-1,3-thiazolidin-3-yl\} 5c. Poudre rouge brique. Rdt: $45 \%$; Pf: $214^{\circ} \mathrm{C} ; \mathrm{R}_{\mathrm{f}}$ (CHCl3/MeOH, 9/1): 0.42; (MeOH) $\lambda \max : 422 \mathrm{~nm} ; \mathrm{IR}(\mathrm{KBr}) \mathrm{cm}^{-1}:$ 3410-2501(OHacide), 1730(C=O), $1690(\mathrm{C}=\mathrm{O}), 1601(\mathrm{C}=\mathrm{N}), 1201(\mathrm{C}=\mathrm{S}) ; \mathrm{RMN}^{1} \mathrm{H}\left(250 \mathrm{MHz}, \mathrm{DMSO}_{\mathrm{d}-6}\right) \delta \mathrm{ppm}: 2.28$ $(\mathrm{d}, 3 \mathrm{H}, \mathrm{J}=, 1.25 \mathrm{H}), 4.34(\mathrm{~s}, 2 \mathrm{H}), 6.68(\mathrm{q}, 1 \mathrm{H}, \mathrm{J}=1.25 \mathrm{~Hz}), 6.86-7.42(\mathrm{~m}, 4 \mathrm{Har}), 8.86(\mathrm{~s}, 1 \mathrm{H}$, 
$\mathrm{H}-\mathrm{C}=\mathrm{N}) ; \quad \mathrm{RMN}^{13} \mathrm{C} \quad\left(62.5 \mathrm{MHz}, \quad \mathrm{DMSO}_{\mathrm{d}-6}\right) \quad \delta$ ppm: $13.46,\left(\mathrm{CH}_{3}\right), \quad 46.73\left(\mathrm{CH}_{2}\right), 82.11$, 104.77(C C $_{5}$ thiazol), $113.45,122.38, \quad 126.28, \quad 134.9, \quad 146.96, \quad 149.41, \quad 150.12$, $156.67,163.63$ (Car), $167.85(\mathrm{C}=\mathrm{O}), 189.06(\mathrm{C}=\mathrm{S})$.

Acide\{(5E)-5-[3-\{[(E)-furan-2-ylméthylidéne]amino\}-4-méthyl-1,3-thiazol-2(3H)-ylidéne]-4 -oxo-2-thioxotetrahydrothiophen-3-yl\} 5d.Poudre rouge. Rdt: $48 \%, \quad \mathrm{Pf}: 218^{\circ} \mathrm{C}, \mathrm{R}_{\mathrm{f}}$ (CHCl3/MeOH, 9/1) : 0.65 ; (MeOH) $\lambda \max : 420 \mathrm{~nm}$; IR (KBr) $\mathrm{cm}^{-1}: 3410-2501(\mathrm{OHacide})$, $1730(\mathrm{C}=\mathrm{O}), \quad 1695(\mathrm{C}=\mathrm{O}), \quad 1612(\mathrm{C}=\mathrm{N}), \quad 1212(\mathrm{C}=\mathrm{S}) . \quad \mathrm{RMN}^{1} \mathrm{H} \quad(250 \mathrm{MHz}, \quad$ DMSOd-6): $\delta$ ppm ;2.28 (d,3H, J $\left.{ }^{4}=, 1.00 \mathrm{~Hz}, \mathrm{CH}_{3}\right), 2.94\left(\mathrm{~s}, 2 \mathrm{H}, \mathrm{CH}_{2} \mathrm{CO}\right), 6.91\left(\mathrm{q}, 1 \mathrm{H}, \mathrm{J}^{4}=1.00 \mathrm{~Hz}, \mathrm{H}_{5}\right.$ thiazol), 7.74-7.42(m, 4Har), $8.22(\mathrm{~s}, 1 \mathrm{H} ; \mathrm{OH}), 9.04(\mathrm{~s}, 1 \mathrm{H}, \mathrm{H}-\mathrm{C}=\mathrm{N})$.

Acide\{(5E)-5-[3-\{[(E)-(3-hydroxy-4-méthoxyphényl)méthylidéne ]amino\}-4-méthyl-1,3-thiaz ol-2(3H)-ylidéne]-4-oxo-2-thioxo-1,3-thiazolidin-3-yl\} 5e. Poudre mauve. Rdt: 66 \%, Pf: $205^{\circ} \mathrm{C} ; \mathrm{R}_{\mathrm{f}(\mathrm{CHCl} 3 / \mathrm{MeOH}, 9 / 1)}: 0.5$; (MeOH) $\lambda \max : 430 \mathrm{~nm} ; \mathrm{IR}(\mathrm{KBr}) \mathrm{cm}^{-1}: 3391-2520(\mathrm{OH}$ acide $), 1720(\mathrm{C}=\mathrm{O}), 1624(\mathrm{C}=\mathrm{O}), 1598(\mathrm{C}=\mathrm{N}), 1201(\mathrm{C}=\mathrm{S}) ; \quad \mathrm{RMN}^{1} \mathrm{H}\left(250 \mathrm{MHz}, \mathrm{DMSO}_{\mathrm{d}-6}\right)$ $\delta$ ppm : $2.21\left(\mathrm{~d}, 3 \mathrm{H}, \mathrm{J}=1.00 \mathrm{~Hz}, \mathrm{CH}_{3}\right), 2.98\left(\mathrm{~s}, 3 \mathrm{H}, \mathrm{CH}_{3} \mathrm{O}\right), \quad 4.45\left(\mathrm{~s}, 2 \mathrm{H}, \mathrm{CH}_{2} \mathrm{CO}\right), 6.90(\mathrm{q}, 1 \mathrm{H}$, $\left.\mathrm{J}^{4}=1.00 \mathrm{~Hz}\right), \quad 7.41-7.80(\mathrm{~m}, 3 \mathrm{Har}), \quad 9.11(\mathrm{~s}, 1 \mathrm{H}, \mathrm{H}-\mathrm{C}=\mathrm{N}), 10.41(\mathrm{sl}, 1 \mathrm{H}, \mathrm{OH})$.

\section{CONCLUSION}

Nous avons décrit la synthèse de nouvelles bases de Schiff chromophores $\mathbf{5}$. Tous les composés synthétisés ont été caractérisés par les méthodes spectroscopiques IR, RMN proton et carbone 13. Les bases de Schiff chromophores $\mathbf{5}$ sont obtenues après trois étapes, à partir d'une molécule chef de file, l'aminothione 1. L'aminothione et la rhodanine acétique ont été préparés à partir de réactif usuels de laboratoire. L'utilisation de l'analyse spectroscopique UV-Vis a permis de déterminer les longueurs d'ondes maximums. Nous avons constaté le phénomène des électrons $\pi$ délocalisés, responsable de l'effet bathochrome, lorsque l'on passe des iminothiones 2a-e aux bases de Schiff 5a-e. Les bases de Schiff 5 obtenues sont de nouveaux colorants pi-conjugués, solubles dans la plupart des solvants organiques et possédant des groupes chromophores leur permettant d'absorber les photons solaire grâce à une fonction d'ancrage sur la surface des oxydes métalliques. 


\section{REFERENCES}

[1] Green M.A, Emery K, King D.L, Igari S, WartaW. Solar cell efficiency tables (version 22). Prog. Photovolt.: Res. Appl. 2003, 11, 347-352, doi: 10.1002/pip.499

[2] Insuasty, A, Ortiz A, Tigreros, A, Solarte E, Insuasty B, N. Martin N. 2-(1, 1-Dicyano methylene) rhodanine A novel, efficient electron acceptor. Dyes Pigm, 2011, 88(3),385-390, doi: 10.1016/j.dyepig.2010.08.011

[3] Pushkara Rao V, Jen A.K.-Y, Caldwell J.B. Rhodanine-methine as p electron acceptor in second-order nonlinear optical chromophores.; Tetrahedron Lett., 1994, 35(23), 3849-3852, doi:10.1016/S0040-4039(00)76683-4

[4] Ray J, Panja N, Nandi P.K, Martin J.J, Jones Jr W E. Spectroscopic and ab initio study of an intramolecular charge transfer (ICT) rhodanine derivative. J. Mol. Struct, 2008, 874, 121-127, doi:10.1016/j.molstruc.2007.03.044

[5] Zhou Y, Xiao M, Liu W, Du Z, Chen W, Ouyang D, Han L, Wana X, Yang R. Synthesis and photovoltaic properties of new small molecules with rhodanine derivative as the end- cap ped blocks.Organic Electronics., 2015, 17, 355-363 , doi : 10.1016/j.orgel.2014.12.031

[6] Qian, X, Lu L, Zhu Y-Z, Gao H-H, Zheng J-Y. Triazatruxene-based organic dyes containing a rhodanine-3-acetic acid acceptor for dye-sensitized solar cells.; Dyes and Pigments, 2015, 113, 737-742, doi:10.1016/j.dyepig.2014.10.007

[7] Morel D. L, Ghosh A. K, Feng T, Stogryn E- L, Purwin P. E, Shaw R. F, Fishman. Highefficiency organic solar cells. C.Appl. Phys. Lett. 1978, 32, 495-497, doi: 10.1063/1.90099 [8] O’Regan B, Grätzel M. A low-cost, high-efficiency solar cell based on dye-sensitized colloidal $\mathrm{TiO}_{2}$ films. ; Nature 1991, 353, 737-740, doi: 10.1038/353737a0

[9] a) Nazeeruddin M. K, Kay A, Rodicio I, Humphry-Baker R, Müller E, Liska P, Vlachopoulos N, Grätzel M. Conversion of Light to Electricity bycis-Xz Bis ( 2,2'-bipyridyl -4, 4'-dicarboxylate) ruthenium (II) Charge-Transfer Sensitizers ( $\mathrm{X}=\mathrm{C} 1-, \mathrm{Br}-, \mathrm{I}-, \mathrm{CN}-$, andSCN-) on Nanocrystalline Ti02 Electrodes. J. Am. Chem. Soc. 1993, 115, 6382-6390, doi: 10.1021/ja00067a063 b) Grätzel M, Recent Advances in Sensitized Mesoscopic Solar Cells. Acc. Chem.Res. 2009, 42(11), 1788-1798, doi: 10.1021/ar900141y c) Hagfeldt A, Boschloo G, Sun L, Kloo L, Pettersson H, Dye-Sensitized Solar Cells. Chem. Rev.2010,110, 
6595-6663, doi: 10.1021/cr900356p

[10] Ito S, Zakeeruddin SM, Humphry-Baker R, Liska P, Charvet R, Comte P, Nazeeruddin M K, Péchy P, Takata M, Miura H, Uchida S, Grätzel M. High-efficiency organic-dye-sensitized solar cells controlled by nanocrystalline- $\mathrm{TiO}_{2}$ electrode thickness. Adv Mater 2006, 18, 1202-120 5, doi: 10.1002/adma.200502540

[11] Ito S, Miura H, Uchida S, Takata M, Sumioka K, Liska P, Comte P, Péchy P, Grätzel M. High-conversion efficiency organic dye-sensitized solar cells with a novel indoline dye. Chem Commun., 2008, 5194-5196, doi: 10.1039/B809093A

[12] Abou-Dobara M.I, El-Sonbati A.Z, Morgan Sh M. Influence of substituent effects on spectroscopic properties and antimicrobial activity of 5-(4'-substituted phenylazo)-2-thioxothiazolidinone derivatives.; World Sh.M. J. Microbiol. Biotechnol. 2013, 29(1), 119-126.

[13] a) Jin X., Synthesis and antimicrobial evaluation of L-phenylalanine-derived C5- substitu ted rhodanine and chalcone derivatives containing thiobarbituric acid or 2-thioxo-4- thiazolidi none. Eur.J.of Med.Chemistry, 2012, 56, 203-209, doi:10.1016/j.ejmech.2012.08.026 b) Tomasic T, Masic L. P. Rhodanine as a privileged scaffold in drugdiscovery. Curr. Med. Chem. 2009, 16, 484-494, doi: 10.2174/092986709788186200

[14] El-Sonbati, A.Z. El-Bindary, A.A. El-Dissouky, A. El-Gogary, T.M. Hilali, A.S. Substituents effect onthe spectral studies on ruthenium(III) complexes of 5(-4'-derivatives phenyldiazo)-3-phenyl-2-thioxo-4-thiazolidinone. Spectrochimica Acta Part A: Molecular and Biomolecular Spectroscop 2002, 58,1623-1629, doi:10.1016/S1386-1425(01)00627-8

b) El-Bindary A.A, El-Sonbati A.Z, Diab M.A., Morgan Sh.M. Geometrical structure, potentiometric and thermodynamic studies of rhodanine azodye and its metal complexes. Journal of Molecular Liquids., 2015, 201, 36-42, doi: 10.1016/j.molliq.2014.11.022

[15] Humphlett W. J, Lamon R. W, 4-Thiazoline-2-thiones. I. The Structure of Intermediate 4-Hydoxythiazolidine-2-thiones.; J. Org. Chem., 1964, 29, 2146- 2148, doi: 10.1021/jo 0103 $1 \mathrm{a} 008$ 


\section{How to cite this article:}

Kasmi-Mir S, ElKebich M, Dembahri Z, Haouzi A and Kirsch G. Synthesis of new schiff bases as materials for the design of photovoltaics cells. J. Fundam. Appl. Sci., 2016, 8(2), 569-583. 